\title{
Diaphragmatic Muscle Fiber Type Development in Swine
}

\author{
DENNIS E. MAYOCK, JOHN HALL, JON F. WATCHKO, THOMAS A. STANDAERT, AND \\ DAVID E. WOODRUM \\ Division of Neonatal and Respiratory Diseases, Department of Pediatrics, University of Washington, School of \\ Medicine, Seattle, Washington 98195
}

\begin{abstract}
Diaphragmatic muscle fiber types were determined in the costal and crural segments of swine diaphragm at 4 postnatal ages (1 day, 1 month, 6 months, and between 3-6 yr of age). Fiber types were differentiated by enzyme histochemistry for adenosine triphosphatase and reduced nicotinamide adenine dinucleotide. A progressive increase in the number of type I fibers occurred in both costal and crural segments from birth to 6 months of age. The number of type IIA fibers decreased and type IIB fibers increased over the same time period. Type IIC fibers were present through 1 month of age, were rarely observed at 6 months, and were not found in older animals. Type I fibers were more numerous in the crural portion of the diaphragm. The cross-sectional area of all fiber types in both costal and crural segments increased significantly with age. No preferential fiber type growth was noted in either segment of the diaphragm. These data suggest that the pig diaphragmatic muscle is differentiated into its adult form by 6 months of postnatal age, but fiber cross-sectional area growth continues along with body growth. (Pediatr Res 22: 449-454, 1987)
\end{abstract}

\section{Abbreviations}

\section{ATPase, adenosine triphosphatase}

NADH-TR, reduced nicotinamide adenine dinucleotide tetrazolium reductase

Respiratory failure is a common problem in the neonate and one that accounts for significant mortality and morbidity during this early stage in life. The pathophysiologic mechanisms that lead to respiratory failure and the need for ventilatory support are not clearly defined. The major muscle of respiration, the diaphragm, has been the focus of intensive investigation recently in an attempt to define these mechanisms. However, most studies have utilized adult animals and humans to elucidate the causes of respiratory failure $(1-8)$. Little attention has been focused on the neonate. In adult humans and animals, the response of certain muscle groups to various stresses can be related to their fiber type composition $(2,9-11)$.

The newborn piglet has been used extensively in our laboratory as a model in which to study diaphragmatic muscle function in a developmental context (12-17). The normal pattern of peripheral skeletal muscle development in the pig that occurs with postnatal maturation has been described $(18-23)$. However, the

Received January 21, 1987; accepted May 18, 1987.

Correspondence Dennis E. Mayock, M.D., Pediatrics RD-20, University of Washington, Seattle, WA 98195

Supported in part by Pediatric Pulmonary Training Grant MCJ 000955 and by Pediatric Pulmonary SCOR Grant HL 19187 pattern of respiratory muscle fiber type development has not been delineated. We examined the fiber type distribution and cross-sectional area in the swine diaphragm during postnatal development from the neonate to the adult in an effort to provide anatomic correlates for physiologic studies of diaphragmatic function in the developing piglet.

\section{MATERIALS AND METHODS}

Tissue sampling. Specimens of the left costal and crural diaphragm from four Yorkshire pigs were obtained at four postnatal ages. Two male and two female animals were studied at 1 day, 1 month, and 6 months of age. Four sows were studied at 3-6 yr of age. Samples were taken immediately following sacrifice with intraperitoneal pentobarbital overdose. The diaphragm was rapidly exposed and muscle was sampled from the left anteriolateral portion of the costal diaphragm midway between the central tendon and the rib insertion. Samples of the left crural diaphragm were obtained from the midportion of the muscle belly. The sample sizes were approximately $1 \times 3 \times 3 \mathrm{~mm}$. The muscle samples were mounted on cork at approximately resting length and quick frozen in isopentane cooled in liquid nitrogen. They remained frozen at minus $70^{\circ} \mathrm{C}$ until sectioned.

Histochemistry. Frozen specimens were brought to a temperature of minus $20^{\circ} \mathrm{C}$ and serial $10-\mu \mathrm{m}$ cross-sections were cut on an American Optical cryostat (American Optical Corporation, Buffalo, NY). Sections were dried at room temperature for $30-$ $60 \mathrm{~min}$ and then incubated in a battery of histochemical media. Samples of human peripheral skeletal muscle obtained from infants and adults for other studies were incubated simultaneously and served as controls for the histochemical reactions. Muscle samples from the day-1, 1-month, and 6-month-old swine were batched for staining. All samples that visually demonstrated unacceptable sectioning or histochemical staining were discarded and repeated. Additionally, if unacceptable staining was found in the internal controls, the swine samples were discarded and repeated. Samples from the adult swine were incubated in the histochemical media simultaneously with control adult human muscle samples. Reactions were performed to demonstrate the activities of NADH-TR and myofibrillar ATPase. The NADH-TR assay was performed according to the method of Dubowitz and Brooke (24). Myofibrillar ATPase assay was performed as described by Chayen et al. (25). Serial sections were preincubated at alkaline $\mathrm{pH}(10.3)$ and at acid $\mathrm{pH}(4.5$ and 4.3) prior to the myofibrillar ATPase assay (24). The $\mathrm{pH}$ of the incubation solutions was adjusted at $37^{\circ} \mathrm{C}$.

Fibers were visually typed according to the schema in Figure 1. Initially, the fibers were classified as either type I or II by their ATPase staining intensity following preincubation at a $\mathrm{pH}$ of 10.3. Type I fibers demonstrate no reaction at this $\mathrm{pH}$ while type II fibers stain intensely. Type II fibers were then subgrouped by their ATPase differential staining reaction following preincuba- 
tion at pH 4.3 and 4.5 . It was not possible to subgroup type II fibers utilizing the NADH-TR assay as all fibers stained intensely by this method on day 1 of life as previously documented (1821, 23) (see Fig. 2). Similar staining occurred in most specimens at 1 month of age (five of eight costal specimens). Therefore, serial sections were compared for differential staining by the ATPase assay. The NADH-TR and ATPase assays were utilized for differential fiber typing in the specimens of diaphragm obtained at 6 months and 3-6 yr. Drawings of microscopic projections of the muscle sections were made utilizing a Zeiss micro-

\begin{tabular}{|c|c|c|c|c|}
\hline & \multicolumn{2}{|c|}{ Muscle } & \multicolumn{2}{|c|}{ er Type } \\
\hline & 1 & $2 \mathrm{~A}$ & $2 B$ & $2 \mathrm{C}$ \\
\hline ATP-ase & & & & \\
\hline Preincubated $\mathrm{pH} 10.3$ & & & & \\
\hline$\overline{A T P}$ - ase & & & & \\
\hline Preincubated $\mathrm{pH} 4.5$ & & & & \\
\hline ATP-ase & & & & \\
\hline Preincubated $\mathrm{pH} 4.3$ & & & & \\
\hline NADH - TR & & & & \\
\hline
\end{tabular}

Fig. 1. Histochemical reaction intensities in swine diaphragm Scheme for classification of muscle fibers according to relative reaction intensity. See "Materials and Methods" for further details. scope and drawing tube (Carl Zeiss, Inc., Thornwood, NY) and identical fields from serial sections of the muscle specimen were overlaid on the drawing to allow for subgrouping of the fiber types. Between 127 and $742($ mean $=453)$ fibers were traced per tissue sample.

Cross-sectional area. The cross-sectional area of each fiber was determined utilizing a Numonics digitizing tablet and electronic graphics calculator model 2400 (Numonics Corporation, Lansdale, PA). Fiber outlines were traced on the drawings previously

Table 1. Diaphragm muscle fiber type composition*

\begin{tabular}{ccccc}
\hline & Type I & Type IIA & Type IIB & Type IIC \\
\hline & $\%$ & $\%$ & $\%$ & $\%$ \\
Costal & & & & \\
Day 1 & $18 \pm 2$ & $61 \pm 5$ & $10 \pm 2$ & $11 \pm 2$ \\
1 mo & $29 \pm 3$ & $56 \pm 4$ & $3 \pm 1$ & $13 \pm 3$ \\
6 mo & $49 \pm 4$ & $31 \pm 4$ & $20 \pm 7$ & $1 \pm 1$ \\
$3-6$ yr & $48 \pm 3$ & $24 \pm 6$ & $28 \pm 4$ & 0 \\
& & & & \\
Crural & & & & \\
Day 1 & $20 \pm 1$ & $61 \pm 2$ & $9 \pm 1$ & $10 \pm 2$ \\
1 mo & $39 \pm 2$ & $43 \pm 3$ & $3 \pm 1$ & $15 \pm 1$ \\
6 mo & $55 \pm 3$ & $30 \pm 5$ & $13 \pm 4$ & $2 \pm 1$ \\
3-6 yr & $58 \pm 5$ & $20 \pm 4$ & $23 \pm 7$ & 0 \\
\hline
\end{tabular}

$* n=4$ for each group. Percentage of each fiber type given as the mean \pm SEM. The total percent of fibers may exceed $100 \%$ due to rounding error. See text for further details.
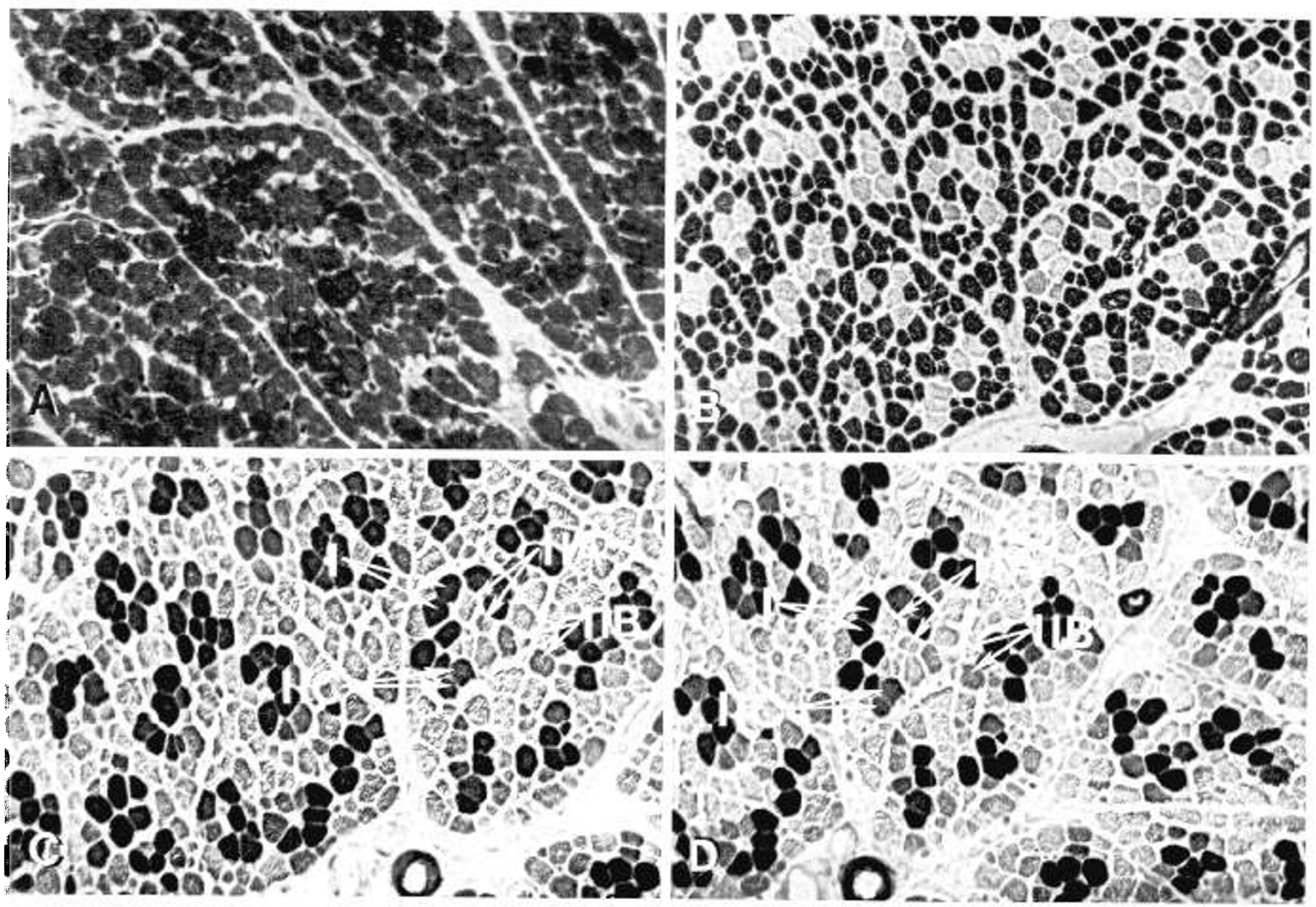

Fig. 2. Muscle fiber histochemical reactions on day 1 in swine. Representative costal diaphragm muscle sample on day 1 of life. NADH-TR and ATPase histochemical reactions. Original magnification is $\times 64 . A, \mathrm{NADH}-\mathrm{TR}$ : all fibers stain intensity precluding fiber subtyping. $B, \mathrm{ATP}$ ase at $\mathrm{pH}$ 10.3: Dark fibers are type II, light staining fibers are type I. $C$, ATPase at $\mathrm{pH}$ 4.5: representative fibers are labeled. D, ATPase at pH 4.3: representative fibers are labeled. 
made from the ATPase reaction slide preincubated at $\mathrm{pH} 10.3$ and the area determined by the Numonics calculator was converted to square microns utilizing a micrometer slide as a standard at a specific magnification. The area of 50 fibers in each fiber type subgroup in each sample were determined if possible.

Results were analyzed by analysis of variance for fiber type or cross-sectional area versus age and location utilizing Minitab (Release 82.1, 1984, PC version).

\section{RESULTS}

Table 1 lists the fiber type composition of the two segments of the diaphragm at the four study ages. Representative sections from day-1 (Fig. 2), 1-month-old (Fig. 3), and 6-month and 3to 6-yr old animals (Fig. 4) are included. No significant differences were noted between the costal and crural segments for type II fibers at any specific age. A significant progressive increase in type I fibers in both segments of the diaphragm was noted until 6 months of age $(p<0.001)$. No further increase was noted in the specimens from animals at 3-6 yr of age. Type I fibers were more numerous in the crural compared to the costal diaphragm $(p<0.02)$. The quantity of type IIA fibers progressively decreased in both segments of the diaphragm through 6 months $(p<$ $0.001)$. Type IIB fiber quantity averaged $10 \%$ at birth, appeared to decrease slightly at 1 month of age, then increased in both segments of the diaphragm to 3-6 yr $(p<0.001)$. A small proportion of type II fibers did not demonstrate acid reversal and were classified as type IIC. The percent of type IIC fibers decreased as the animals matured, were rarely seen at 6 months, and were not observed at 3-6 yr of age.
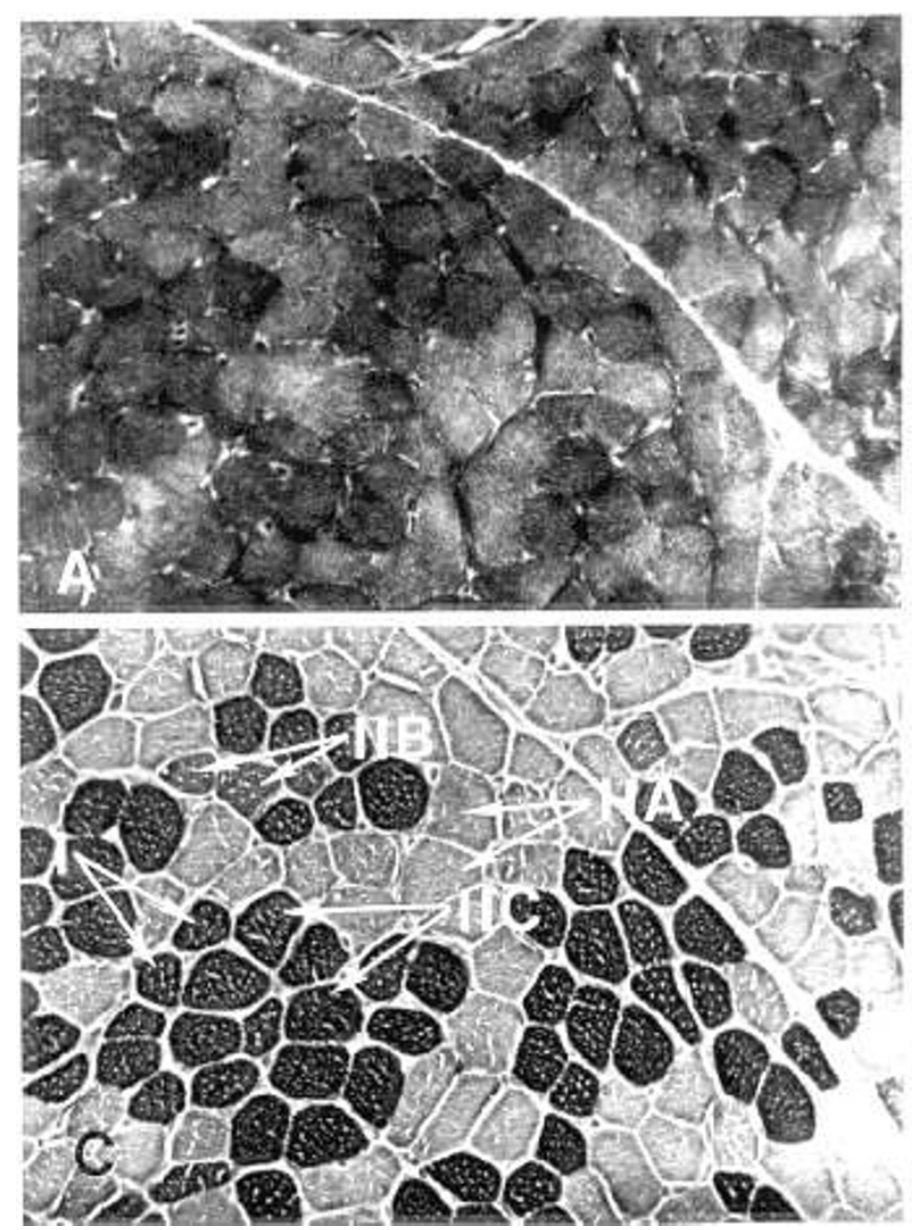

All fibers significantly increased in cross-sectional area with postnatal maturation (Fig. 4; Tables 2 and 3). No differences in area were found between the costal and crural segments of the diaphragm for any fiber type subgroup at each age. On day 1 of life, the fibers were round in shape (Fig. 2). The profile of type I fibers remained round with age whereas type II fibers became larger and more polygonal with maturation (Fig. 4).

\section{DISCUSSION}

The normal postnatal changes in fiber types and cross-sectional area of the costal and crural diaphragmatic muscle in the piglet have been determined. Type I fiber quantity was found to increase with maturation to 6 months of age. Type IIA fiber quantity decreased through 6 months of age while type IIB fiber quantity increased. Type IIC fibers comprised approximately $10 \%$ of all fibers at birth, were rarely seen by 6 months, and were not found in the adult pig. All fibers demonstrated a significant increase in cross-sectional area that continued to the 3- to 6-yr group of animals.

Critique. Fiber type determination by histochemistry is a standardized methodology when used to examine specimens of mature muscle $(2-4,7,8,10,24)$. However, questions have been raised in regards to the applicability of this methodology to developing muscle in the fetus and newborn (26). Guth and Samaha (26) demonstrated a discrepancy between ATPase histochemical staining of newborn rabbit muscle and the biochemical activity of the ATPase enzyme. Additionally, Guth (27) found that the histochemical demonstration of myofibrillar ATPase activity could be misleading since the reaction product may
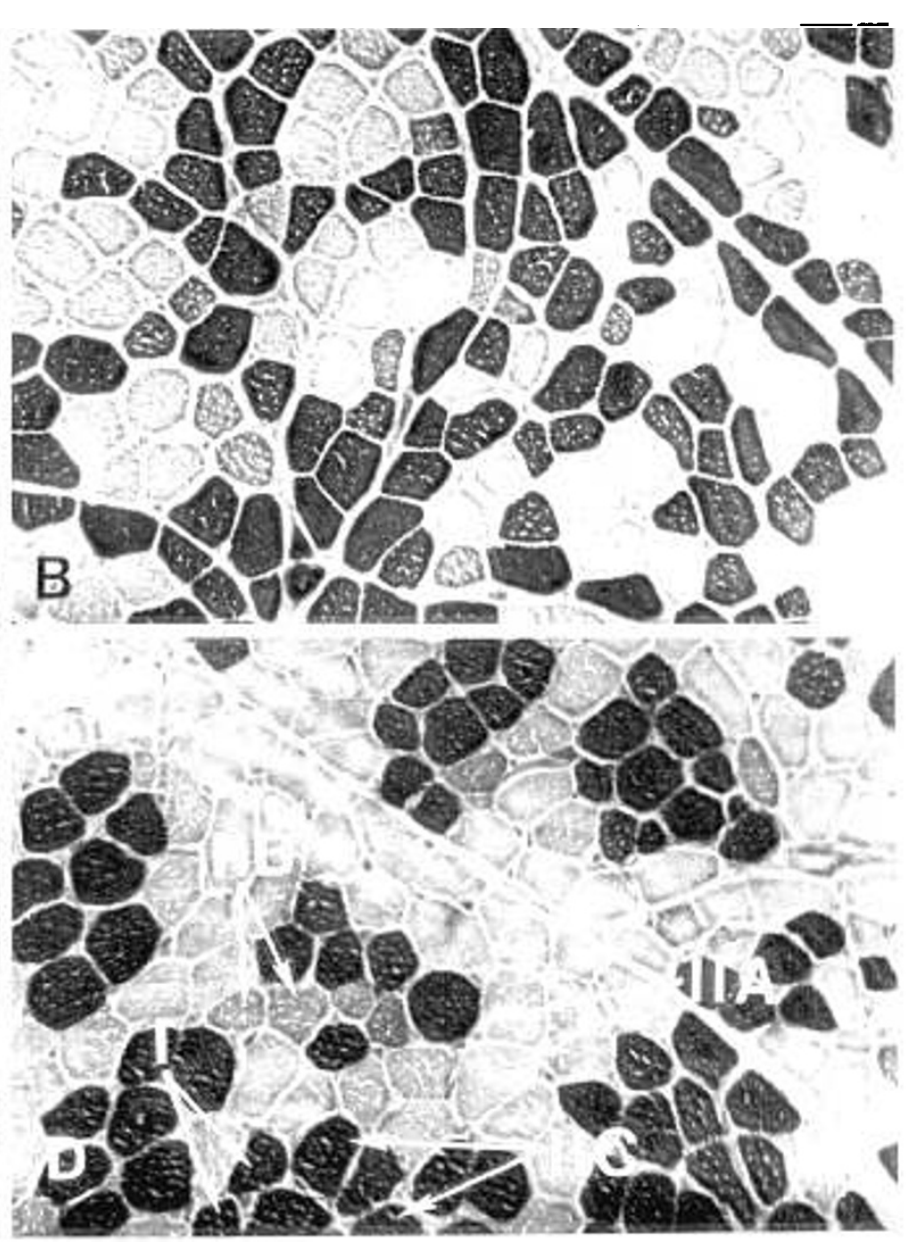

Fig. 3. Muscle fiber histochemical reactions at 1 month in swine. Representative costal diaphragm muscle sample at 1 month of life. NADH-TR

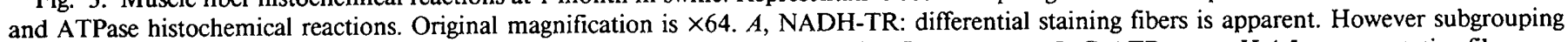

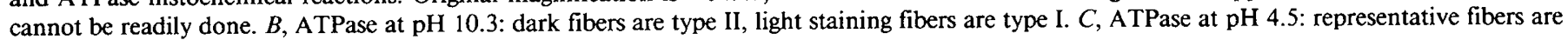
labeled. $D$, ATPase at $\mathrm{pH}$ 4.3: representative fibers are labeled. 


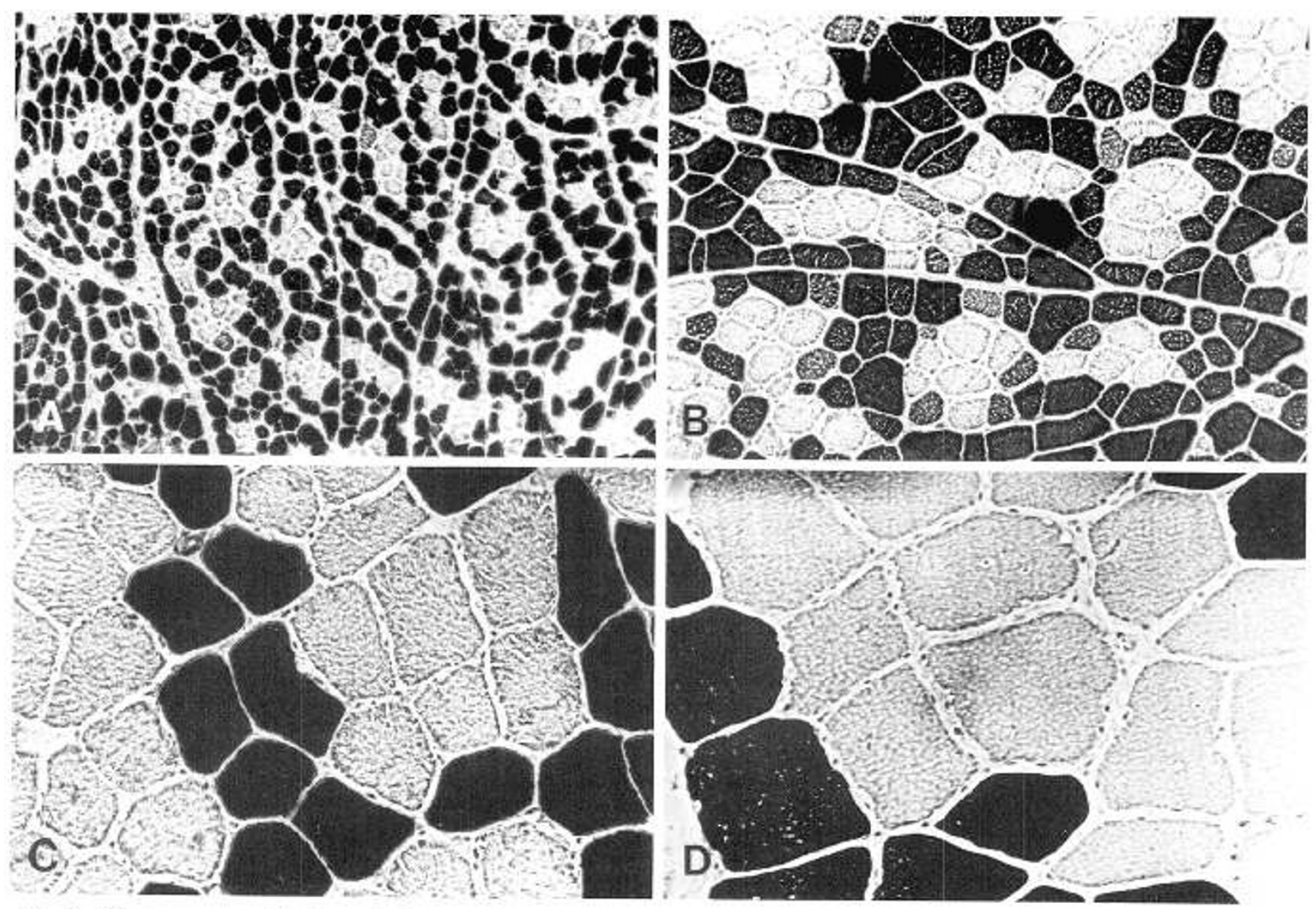

Fig. 4. Fiber type cross-sectional area development in swine. Representative costal diaphragm muscle samples at the four different ages studied. ATPase histochemical reactions following preincubation at $\mathrm{pH} 10.3$. Original magnification is $\times 64$. $A$, day $1 ; B, 1$ month; $C, 6$ months; $D, 4$ yr.

Table 2. Fiber cross-sectional area*

\begin{tabular}{lcccccccc} 
& \multicolumn{7}{c}{ Costal diaphragm } \\
\cline { 2 - 8 } & Type I & $n$ & Type IIA & $n$ & Type IIB & $n$ & Type IIC & $n$ \\
\hline Day 1 & $138 \pm 14$ & 59 & $204 \pm 57$ & 61 & $150 \pm 30$ & 49 & $139 \pm 20$ & 55 \\
1 mo & $656 \pm 109$ & 50 & $733 \pm 108$ & 50 & $441 \pm 62$ & 13 & $453 \pm 78$ & 41 \\
6 mo & $4387 \pm 464$ & 50 & $4275 \pm 451$ & 50 & $5413 \pm 478$ & 41 & $4211 \pm 1076$ & 4 \\
3-6 yr & $6862 \pm 539$ & 50 & $5038 \pm 396$ & 42 & $5962 \pm 1170$ & 50 & & 0 \\
\hline
\end{tabular}

* Area is given in $\mu^{2}$. The mean and SE of the cross-sectional area are given for each age group and fiber type. $n$ is the mean number of fibers of a specific type counted per animal. All fiber types significantly increased in cross-sectional area (two-way analysis of variance, $p<0.001$ ).

Table 3. Fiber cross-sectional area*

\begin{tabular}{lcccccccc} 
& \multicolumn{9}{c}{ Crural diaphragm } \\
\cline { 2 - 9 } & Type I & $n$ & Type IIA & $n$ & Type IIB & $n$ & Type IIC & $n$ \\
\hline Day 1 & $119 \pm 13$ & 50 & $145 \pm 10$ & 51 & $125 \pm 7$ & 47 & $102 \pm 7$ & 42 \\
1 mo & $568 \pm 39$ & 50 & $624 \pm 35$ & 50 & $452 \pm 10$ & 14 & $366 \pm 28$ & 50 \\
6 mo & $4268 \pm 230$ & 50 & $4236 \pm 169$ & 50 & $5157 \pm 76$ & 38 & $2897 \pm 309$ & 9 \\
$3-6$ yr & $6927 \pm 860$ & 50 & $5855 \pm 911$ & 48 & $6141 \pm 1266$ & 36 & & 0 \\
\hline
\end{tabular}

* Area is given in $\mu^{2}$. The mean and SE of the cross-sectional area are given for each age group and fiber type. $n$ is the mean number of fibers of a specific type counted per animal. All fiber types significantly increased in cross-sectional area (two-way analysis of variance, $p<0.001$ ).

not have originated solely in the myofibrils themselves, but also in mitochondria. However, more recent workers have found good correlations between myofibrillar ATPase staining and oxidative staining over a wide age range in baboon ventilatory muscles (28). Maxwell et al. (28) found that all fibers in the fetal baboon stained intensely for NADH-TR. Similar findings were reported in piglets (18-23) and in kittens (29). These later studies relied on myofibrillar ATPase activity for fiber type classification rather then oxidative enzyme reactions. The histochemical studies in the baboon demonstrating high oxidative capacity of all fibers in the respiratory muscles was in excellent agreement with measures of function (28) and is the first study to correlate diaphragmatic muscle function with histochemical findings within a developmental context. We conclude that the histo- 
chemical methodology utilized in this study accurately identifies muscle fiber types in the newborn piglet.

Suzuki and Cassens (30) found that the histochemical demonstration of ATPase enzymatic activity was very $\mathrm{pH}$ sensitive. These results lead us to utilize the preincubation of $\mathrm{pHs}$ of 10.3 , 4.5 , and 4.3 to carefully differentiate fiber type subgroups and allow for a second check to define type IIB fibers.

Maturational changes in muscle fiber types. Previous studies of postnatal changes in fiber types in the pig have focused primarily on peripheral skeletal muscle (18-23). The developmental changes in muscle fiber type distribution in the pig, both in peripheral skeletal muscle and in the costal diaphragm have been described (18). As in the present study and several previous investigations $(18,19,23)$, all fibers demonstrated high oxidative histochemical activity, as evidenced by NADH-TR enzyme staining, for several weeks after birth. The postnatal developmental changes in fiber type distribution were similar to the present study, but cannot be compared directly to the present study since the age of the piglets studied were not given, tissue preparation was different, and the histochemical techniques were not identical. Suzuki and Cassens (22) studied myofiber type development from birth to $16 \mathrm{wk}$ of age in swine peripheral skeletal muscle. They found a progressive increase in type I fiber quantity with a decrease in type II fibers out to 8 wk of life. However, the quantity of type I fibers in the longissimus, rectus femoris, and masseter muscles was very low compared to diaphragmatic muscle examined in this study. Fiber type distribution found in the diaphragm of our piglets more closely resembled those found in the trapezius muscle examined (22).

In the adult pig, Davies and Gunn (31) examined diaphragmatic muscle fiber type composition and found $45 \%$ type I, $20 \%$ type IIA, and $33 \%$ type IIB fibers, values in close agreement with our data. Similar type I fiber quantities in adult diaphragmatic muscle have been found in the cat $(4,7,8)$ and the rat (3).

Maxwell et al. (28) examined the histochemical properties of baboon respiratory muscles from midgestation to adulthood. In fetal and neonatal muscle, they found a high percentage of type IIC fibers which stained intensely for oxidative enzymes. They concluded that the respiratory muscles of the baboon during gestation and in the neonatal period are high in oxidative capacity. However, the baboon respiratory muscles are substantially more differentiated at birth than in the human. This contrasts with previous studies which suggested that human muscle fiber type differentiation is more advance than in other species (3236).

Keens et al. (37) studied the development of human respiratory muscles from 24 wk gestation to adulthood. They found a progressive increase in type I fiber number in the diaphragm from $10 \%$ in infants less than 37 wk gestation to $25 \%$ in full term infants and $55 \%$ in subjects more than $2 \mathrm{yr}$ of age. Adult levels of type I fibers were reached by 8 months of postnatal age. A similar progression was noted in samples from internal and external intercostal muscles with the exception that the percent of type I fibers was greater at any age and no further differentiation was noted after 2 months of life.

Type I fiber differentiation in swine resembles that found in the human, but with a shifted time frame (37). The findings in the pig at birth are similar to the premature human $<37$ wk gestation; at 1 month, swine are similar to the full-term human infant; swine at 6 months and older are similar to adult humans. When compared to the baboon (28), swine demonstrate a similar pattern of maturational change. However, it would appear that baboon diaphragmatic muscle fiber type differentiation is slightly more advanced at birth than in swine. The newborn pig has a similar fiber type distribution as the baboon at approximately 140 days with the exception that baboon type IIB fibers are not seen until after term. At 3-6 yr, swine have a similar quantity of type I, IIA, and IIC fibers, but less IIB fibers when compared to the baboon.

Maturational changes in fiber cross-sectional area. The values reported by Davies and Gunn (31) for fiber cross-sectional area as determined by planimetry at 10 and 56 days postnatal age would fit with our data for the costal diaphragm. The mean fiber area in their six pigs was $5680 \pm 1170 \mu^{2}$, very similar to those found in the present study. This fiber size was significantly larger then values found in the horse, ox, sheep, dog, cat, rabbit, rat, or mouse. The large area of "myosin-ATPase low" or type I fibers in the costal diaphragm of their pigs and that found in the present study contrasts with previous work that suggested that type I fibers in peripheral skeletal muscle were generally of small crosssectional area probably to allow for adequate substrate delivery (8). Sieck, et al. (8) determined that the type II fibers of the adult cat diaphragm were generally larger then type I fibers, but substantial variability was reported in the cross sectional area of both fiber types.

Bowden and Goyer (38) studied fiber size developmental changes in human diaphragm muscle up to $9 \mathrm{yr}$ of age. They found that diaphragm muscle fibers were larger than other striated skeletal muscle fibers until 9 yr of age. The samples that were obtained at autopsy in preterm and term infants have comparable fiber cross-sectional area to 1-day-old piglets in the present study. However, the piglet fibers were much larger at all succeeding ages as compared to the human.

The results of this study and that of Davies and Gunn (31) would suggest that each diaphragmatic muscle fiber can obtain sufficient substrate to function on an intermittent basis indefinitely despite their large cross-sectional area in the adult.

Relationship of fiber type to function. Hammarberg and Kellerth (39) found that the hindlimb muscles of kittens were quite fatigue resistant and the adult pattern of fatiguability only became evident by 40 to 70 days of postnatal age. This change over in fatigue pattern corresponded very closely to histochemical fiber type maturation (40) and loss of polyneuronal innervation $(41,42)$. Keens et al. (37) concluded that the human neonate is more susceptible to respiratory muscle fatigue based on the large percent of type II fibers present in the respiratory muscles. However, no functional measures were obtained. Maxwell et al. (28) examined the functional properties of baboon respiratory muscles from midgestation to adulthood. Functionally, the diaphragmatic muscle of the premature baboons was found to be more fatigue resistant than that of the adult. They concluded that the respiratory muscles of the baboon during gestation and in the neonatal period are high in oxidative capacity and are fatigue resistant. The finding of moderate to high oxidative activity in the neonatal human diaphragm by the same investigators provides further support for this argument (43).

Lieberman et al. (2) examined the adult guinea pig and human diaphragm. They found an excellent correlation of histochemical fiber type quantities with functional properties in both species. Additionally, they tested human adult males during maximal voluntary ventilation trials and concluded that the initial small decrease in ventilation noted within the first min of the trial was secondary to fatigue of low oxidative fast twitch fibers (or type IIB). The plateau in the maximal voluntary ventilation trial was maintained out to $5 \mathrm{~min}$ and correlated well with the total percent of high oxidative fibers, specifically type I and IIA fibers.

Biochemical measurement of oxidative enzyme activity is necessary for proper interpretation of work capacity of a muscle fiber under specified conditions. Therefore, we can only speculate as to the functional correlates of fiber type subgroups and crosssectional area determined in this study. All fibers demonstrated intense oxidative enzyme histochemical staining early in life (Figs. 2 and 3). If one would consider the sum total of type I and type IIA fibers as being oxidative in nature, then this sum remains high (mean $80 \pm 4 \%$ ) throughout the range of ages studied herein. These findings would suggest that the diaphragm should be relatively fatigue resistant throughout life in the pig. This hypothesis awaits further testing since the diaphragm has not been studied from a functional point of view in the adult pig. However, recent studies in the neonatal piglet have examined 
the functional capabilities of the diaphragm in a developmental context. The force output of the diaphragm increases with postnatal age, body weight, and diaphragmatic wet weight for a given central drive to the muscle (14). Additionally, the neonatal piglet diaphragm can fatigue when confronted with large inspiratory resistive loads despite elevated central drive (12). A decrease in the force frequency curve of the diaphragm at $1 \mathrm{~h}$ of resistive loading occurred indicating fatigue despite the clinical observation that respiration continued, EMG significantly increased, and the spontaneously generated transdiaphragmatic pressure remained elevated. Thus, although fatigue can be uncovered by certain testing procedures, clinical manifestations may not be present.

In summary, the muscle fiber type subgrouping and crosssectional area changes that occur in the costal and crural diaphragm with postnatal maturation in the piglet have been delineated. A progressive increase in the portion of type I fibers was found to 6 months of age. Type I fibers were more numerous in the crural segment compared to the costal segment of the diaphragm. Type IIA fibers decreased and type IIB increased over the same time period. Type IIC fibers represented approximately $10 \%$ of the total fiber quantity at birth, were rarely observed at 6 months of age, and were not found in the 3- to 6-yr old group of animals. Fiber type differentiation was, therefore, completed by 6 months postnatal age. All muscle fibers increased in crosssectional area out to 3-6 yr of age. These results provide correlates for the study of respiratory muscle function in the piglet in a neonatal and developmental context.

Acknowledgments. The authors acknowledge the histopathologic expertise of Dr. Emil Chi. We thank Dr. Albert Berger for his review of the manuscript and the generous use of the $\mathrm{Nu}$ monics digitizing system for cross-sectional area determination.

\section{REFERENCES}

1. Bazzy AR, Haddad GG 1984 Diaphragmatic fatigue in unanesthetized adult sheep. J Appl Physiol 57:182-190

2. Lieberman DA, Faulkner JA, Craig AB, Maxwell LC 1973 Performance and histochemical composition of guinea pig and human diaphragm. J Appl Physiol 34:233-237

3. Metzger JM, Scheidt KB, Fitts RH 1985 Histochemical and physiological characteristics of the rat diaphragm. J Appl Physiol 58:1085-1091

4. Riley DA, Berger AJ 1979 A regional histochemical and electromyographic analysis of the cat respiratory diaphragm. Exp Neurol 66:636-649

5. Roussos C, Fixley M, Gross D, Macklem PT 1979 Fatigue of inspiratory muscles and their synergic behavior. J Appl Physiol 46:897-904

6. Roussos C, Macklem PT 1977 Diaphragmatic fatigue in man. J Appl Physiol 43:189-197

7. Sieck GC, Roy RR, Powell P, Blanco C, Edgerton VR, Harper RM 1983 Muscle fiber type distribution and architecture of the cat diaphragm. J Appl Physiol 55:1386-1392

8. Sieck GC, Sacks RD, Blanco CE, Edgerton VR 1986 SDH activity and crosssectional area of muscle fibers in cat diaphragm. J Appl Physiol 60:12841292

9. Karlsson J, Sjodin B, Jacobs I, Kaiser P 1981 Relevance of muscle fibre type to short intense and prolonged exercise in man. In: Porter R, Whelan $J$ (eds) Human Muscle Fatigue: Physiological Mechanisms. Pitman Medical, London, pp 59-70

10. Burke RE, Levine DN, Tsairis P, Zajac FE 1973 Physiological types and histochemical profiles in motor units of the cat gastrocnemius. $\mathbf{J}$ Physiol (Lond) 234:723-748

11. Thorstensson A, Karlsson J 1976 Fatiguability and fibre composition of human skeletal muscle. Acta Physiol Scand 98:318-322

12. Mayock DE, Badura RJ, Watchko JF, Standaert TA, Woodrum DE 1987 Response to resistive loading in the newborn piglet. Pediatr Res 21:121-125

13. Watchko JF, Mayock DE, Standaert TA, Woodrum DE 1986 Diaphragmatic pressure in piglets: transvenous versus direct phrenic nerve stimulation. Pediatr Pulmonol 2:198-201

14. Watchko JF, Mayock DE, Standaert TA, Woodrum DE 1986 Postnatal changes in transdiaphragmatic pressure in piglets. Pediatr Res 20:658-661

15. Watchko JF, LaFramboise WA, Standaert TA, Woodrum DE 1986 Diaphragmatic function during hypoxemia: neonatal and developmental aspects. J Appl Physiol 60:1599-1604

16. Watchko JF, Standaert TA, Woodrum DE 1987 Diaphragmatic function during hypercapnia: neonatal and developmental aspects. J Appl Physiol 62:768-775

17. Watchko JF, Mayock DE, Standaert TA, Woodrum DE 1987 Effect of inspiratory resistive loading on costal and crural diaphragm electromyograms in piglets. Pediatr Res 21:25-28

18. Davies AS 1972 Postnatal changes in the histochemical fibre types of porcine skeletal muscle. J Anat 113:213-240

19. Beermann DH, Cassens RG, Hausman GJ 1978 A second look at fiber type differentiation in porcine skeletal muscle. J Anim Sci 46:125-132

20. Ashmore CR, Tompkins G, Doerr L 1972 Postnatal development of muscle fiber in domestic animals. J Anim Sci 34:37-41

21. Swatland HJ 1975 Histochemical development of myofibres in neonatal piglets. Res Vet Sci 18:253-257

22. Suzuki A, Cassens RG 1980 A histochemical study of myofiber types in muscle of the growing pig. J Anim Sci 51:1449-1461

23. Cooper CC, Cassens RG, Kastenschmidt LL, Briskey EJ 1970 Histochemical characterization of muscle differentiation. Dev Biol 23:169-184

24. Dubowitz V, Brooke MH 1973 Muscle Biopsy: A Modern Approach. Saunders and $\mathrm{Co}$, Philadelphia

25. Chayen J, Bitensky L, Butcher RG, Poulter LW 1972 A Guide to Practical Histochemistry. Lippincott, Philadelphia, pp 112-115

26. Guth L, Samaha FJ 1972 Erroneous interpretations which may result from application of the myofibrillar ATPase histochemical procedure to developing muscle. Exp Neurol 34:465-475

27. Guth L 1973 Fact and artifact in the histochemical procedure for myofibrillar ATPase. Exp Neurol 41:440-450

28. Maxwell LC, McCarter RJM, Kuehl TJ, Robotham JL 1983 Development of histochemical and functional properties of baboon respiratory muscles. $\mathrm{J}$ Appl Physiol 54:551-561

29. Nystrom B 1968 Histochemistry of developing cat muscles. Acta Neurol Scand 44:405-439

30. Suzuki A, Cassens RG $1980 \mathrm{pH}$ sensitivity of myosin adenosine triphosphatase and subtypes of myofibres in porcine muscle. Histochem J 12:687-693

31. Davies AS, Gunn HM 1972 Histochemical fibre types in the mammalian diaphragm. J Anat 112:41-60

32. Dubowitz V 1965 Enzyme histochemistry of skeletal muscle. I. Developing animal muscle. J Neurol Neurosurg Psychiat 28:516-519

33. Dubowitz V 1965 Enzyme histochemistry of skeletal muscle. II. Developing human muscle. J Neurol Neurosurg Psychiat 28:519-524

34. Farkas-Bargeton E, Diebler MF, Arsenio-Nunes ML, Wehrle R, Rosenberg B 1977 Etude de la maturation histochimique quantitative et ultrastructurale du muscle foetal humain. J Neurol Sci 31:245-259

35. Fenichel GM $1966 \mathrm{~A}$ histochemical study of developing human skeletal muscle. Neurology 16:741-745

36. Beatty CH, Basinger GM, Bocek RM 1967 Differentiation of red and white fibers in muscle from fetal, neonatal, and infant rhesus monkeys. J Histochem Cytochem 15:93-103

37. Keens TG, Bryan AC, Levison H, Ianuzzo CD 1978 Developmental pattern of muscle fiber types in human ventilatory muscles. J Appl Physiol 44:909913

38. Bowden DH, Goyer RA 1960 The size of muscle fibers in infants and children. Arch Pathol 69:188-189

39. Hammarberg C, Kellerth J-O 1975 The postnatal development of some twitch and fatigue properties of single motor units in the ankle muscles of the kitten. Acta Neurol Scand 95:243-257

40. Hammarberg C 1974 The histochemical appearance of developing muscle fibres in the gastrocnemius, soleus and anterior tibial muscles of the kitten, as viewed in serial sections stained for lipids and succinic dehydrogenase. Acta Neurol Scand 50:285-301

41. Bagust J, Lewis DM, Westerman RA 1973 Polyneuronal innervation of kitten skeletal muscle. J Physiol (Lond) 229:241-255

42. Westerman RA, Lewis DM, Bagust J, Edjtehadi G, Pallot D 1973 Communication between nerves and muscles: postnatal development in kitten hindlimb fast and slow twitch muscle. In: Zippel H (ed) Memory and Transfer of Information. Plenum, New York, pp 255-291

43. Maxwell LC, Kuehl TJ, Robotham JL, McCarter RJM 1984 Temporal changes after death in primate diaphragm muscle oxidative enzyme activity. Am Rev Respir Dis 130:1147-1151 